\title{
Parafermions for higher order extensions of the Poincaré algebra and their associated superspace
}

\author{
R. Campoamor-Stursberg*a and M. Rausch de Traubenberg ${ }^{\dagger b}$ \\ ${ }^{a}$ I.M.I-U.C.M, Plaza de Ciencias 3, E-28040 Madrid, Spain \\ ${ }^{b} I P H C-D R S, U d S, C N R S$, IN2P3; 23 rue du Loess, \\ Strasbourg, 67037 Cedex, France
}

July 15, 2009

\begin{abstract}
Parafermions of order two and three are shown to be the fundamental tool to construct superspaces related to cubic and quartic extensions of the Poincare algebra. The corresponding superfields are constructed, and some of their main properties analyzed in detail. In this context, the existence problem of operators acting like covariant derivatives is analyzed, and the associated operators are explicitly constructed.
\end{abstract}

\section{Introduction}

The question whether symmetric and antisymmetric functions are the only physically acceptable types for eigenfunctions can be traced back to the beginning of Quantum Mechanics, and was generally answered in the affirmative until the discovery of new particles and resonances forced to reconsider the possibility of alternative symmetry types.

The first developments on intermediate statistics were developed in 1940, at the same time of Pauli's theorem [1. In this work, the combinatorial method of Bose was used to infer an expression for the average number of particles in a set of states that is independent on the maximal number of particles that occupy a given state. The ansatz was mainly based on the first-quantised formulation of statistics, and therefore this approach was not entirely satisfactory from the quantum field theoretic point of view. In 1953, Green introduced what is nowadays known as paraquantisation [3, 4, leading to two families of generalised statistics, each one containing one of the classical Bose and Fermi statistics types. This pioneering work was later developed and refined by Greenberg and Messiah [5, 6], who settled under which conditions parastatistics do not contradict established experimental facts.

The discovery of the $\Omega^{-}$-hadron in 1964 not only supposed one of the first successes of the symmetry approach to elementary particle classification, to be further worked out in subsequent

\footnotetext{
*e-mail: rutwig@pdi.ucm.es

${ }^{\dagger}$ e-mail: Michel.Rausch@IReS.in2p3.fr
} 
years (finally leading to the Standard Model), but also pointed out some difficulties that were not entirely understood and explained until the overflow of particle discoveries in the 50's and 60's. The quark content of $\Omega^{-}$following from the flavour classification of hadrons apparently meant an evidence to the negative for the Pauli exclusion principle, which reopened the discussion on its range of validity and its exact interpretation with respect to the underlying statistics. This fact, joined to other minor incompatibilities observed earlier, showed that some fundamental characteristics were still to be discovered, and suggested that the newly introduced quarks obeyed not the usual Fermi statistics, but some type of intermediate statistics. Actually this assumption on the para-particle character of quarks provided an alternative solution to the introduction of a threefold degree of freedom for quarks (the colour quantum number) that gave birth to Quantum Chromodynamics (QCD). However, since parastatistics was not amenable to gauging, the idea of paraquantisation as a description of a fundamental symmetry for the quarks was abandoned to the benefit of QCD or more generally of gauge theory.

Subsequently, the gauge principle becomes central in the description of fundamental interactions. This principle together with a series of no-go theorems [7] lead to the dominant framework for a description of physics beyond the Standard Model, namely to supersymmetry or supergravity. Supersymmetry (resp. gauge theories) are based on Lie superalgebras (resp. Lie algebras) which are binary algebras. In spite of the great success of gauge theories and supergravity, one may wonder whether or not some different algebraic structures could play a role in physics, and in particular higher order algebras. This question was rather academic and binary algebras (Lie superalgebras) were dominating the description of the symmetries in particles physics until recently. Indeed, it was realised that higher order algebras could play some roles in physics. For instance, a ternary algebra defined by a fully antisymmetric product appears in the description of multiple M2-branes [8]. Similarly, higher order extensions of the Poincaré algebra were defined without contradicting the no-go theorems [9] and implemented into the Quantum Field Theory (short QFT) frame. Then, despite many efforts, the construction of an adapted "superspace" associated to these latter higher order extensions was not known. The purpose of this paper is to show that parafermions are the basing building block for the construction of a superspace associated to the higher order extensions of the Poincaré algebra considered in [9]. This means that parafermions reappear for the description of symmetries in physics, but in a different context compared to its historical consideration.

In a series of papers, $F$-ary extensions $(F>2)$ of Lie superalgebras, called Lie algebras of order $F$, were introduced and analysed [9, 10, 11, 12. It was then rapidly realised that these new algebraic structures could be used to define higher order extensions of the Poincaré algebra. Among various possibilities, a specific cubic extension of the Poincaré algebra in any space-time dimensions was intensively studied in [13, 14, 15] and implemented into QFT. However, this program to investigate new types of non-trivial extensions of the Poincaré algebra partially failed. Indeed, at present, only invariant free Lagrangians have been constructed. It was even proven that, in four space-time dimensions, for specific types of multiplets, no interaction terms were possible [14]. In order to construct invariant interacting Lagrangians, one of the most promising alternatives would be to identify some adapted " $F$-ary superspace", where the higher order symmetries would be realised by means of differential operators. Such a construction has indeed been considered by several authors in one or two space-time dimensions, where the situation is somewhat exceptional (the Lorentz algebra being either trivial or abelian) [16], and a nice geometrical interpretation was given in [17. It seemed however that the success of such models were deeply related to the low dimension, and as soon as the space-time dimension is higher than three, no corresponding superspace has been identified. This obstruction is certainly due, at least in part, to the type of algebras considered, which are ternary (and in general $F$-ary) instead of binary. 
Among the main difficulties arising in non-binary approaches, we observe that it is not possible to order a given monomial in a definite order, implying that finite dimensional representations are automatically non-faithful [9, 11. Despite these difficulties, it was recently realised that Lie algebras of order $F$ share some similarities with Lie superalgebras. A formal study of Lie algebras of order three gives rise to two interesting results. The first important result in this direction is the construction of groups associated to Lie algebras of order three. Furthermore, the parameters of the transformations have been identified, and correspond to the natural cubic extension of the Grassmann algebra, called the three-exterior algebra (see (3.1) below) introduced by Roby [18. These similarities enable the construction of matrix representations of groups associated to Lie algebras of order three, in terms of matrices, the entries of which belong to the three-exterior algebra [11], in straight analogy with Lie supergroups.

Since the parameters of the transformations generate the three-exterior algebra, it is natural to postulate that these variables generate also the superspace corresponding to the ternary extensions of the Poincaré algebra we are considering. Imposing that a differential realisation of the algebra is obtained by means of the new variables and some associated differential operator, automatically leads to a parafermionic algebra [3, 4]. It is very interesting to notice that two different structures, which have a priori no relation, can be unified by this ansatz. The question whether these two structures (parafermions and Lie algebras of order $F$ ) have some hidden relations arises at once.

The contents of this paper is the following. In section 2. the mean features of Lie algebras of order $F(F>1)$ are recalled together with some emphasis on the cubic and quartic extensions of the Poincaré algebra relevant in the sequel. In section 3. it is shown that parafermions (of order two for ternary extensions and of order three for quaternary extensions) are the fundamental objects to define a superspace associated to the cubic/quartic extension of the Poincaré algebra considered. It is also shown, studying quartic extensions in any space-time dimensions that the case $D=1+9$ is very special. In this particular case a quaternary superspace can be constructed using usual fermions. Moreover, it is interesting to notice that this quartic extension presents some analogy with type IIA supersymmetry. Section 4 is devoted to the study of two types of superfields, as well as the construction of certain operators which can be interpreted as a covariant derivative. It turns out that the implications of these operators for cubic and quartic extensions differ in some fundamental aspects. Some conclusions on these constructions are drawn in section 5.

Finally, we mention for completeness that there has been also some revival of interest for parafermions and parabosons, in a rather different context. In [19] it was realised that paraquantisation is related to Lie superalgebras, and in [20] parafermions were the basic building block to construct some parafermionic extensions of the Poincaré algebra in the context of para-superalgebras. In [21, it was shown that some purely parabosonic (parfermionic) systems are described by a hidden nonlinear (polynomial) supersymmetric quantum mechanics.

\section{Lie algebras of order three}

In this section, we recall the basic properties of Lie algebras of order $F>2$. We also recall the main features of cubic and quartic extensions of the Poincaré algebra that will be relevant in the sequel. Higher order algebraic structures, called Lie algebras of order $F$ and generalising Lie (super)algebras were introduced in [9]. Complex and real Lie algebras of any order $F>2$ may be defined. In this paper we study more precisely elementary real Lie algebras of order three and four. An elementary (real) Lie algebra of order $F$ is given by $\mathfrak{g}=\mathfrak{g}_{0} \oplus \mathfrak{g}_{1}=\left\langle X_{i}, i=1, \cdots, \operatorname{dim} \mathfrak{g}_{0}\right\rangle \oplus$

$\left\langle Y_{a}, a=1, \cdots, \operatorname{dim} \mathfrak{g}_{1}\right\rangle$ where $\mathfrak{g}_{0}$ is a real Lie algebra and $\mathfrak{g}_{1}$ is a real representation of $\mathfrak{g}_{0}$, satisfying 
the following brackets

$$
\begin{aligned}
{\left[X_{i}, X_{j}\right] } & =f_{i j}{ }^{k} X_{k}, \quad\left[X_{i}, Y_{a}\right]=R_{i a}{ }^{b} Y_{b}, \\
\left\{Y_{a_{1}}, \cdots, Y_{a_{F}}\right\} & =\sum_{\sigma \in S_{F}} Y_{a_{\sigma(1)}} \cdots Y_{a_{\sigma(F)}}=Q_{a_{1} \cdots a_{F}}{ }^{i} X_{i},
\end{aligned}
$$

(where $S_{F}$ is the group of permutations of $F$ elements) and fulfilling the following Jacobi identities for any $Y_{a_{1}}, \cdots, Y_{a_{F+1}}$ in $\mathfrak{g}_{1}$

$$
\sum_{i=1}^{F+1}\left[\left\{Y_{a_{1}}, \cdots, Y_{a_{i-1}}, Y_{a_{i+1}}, \cdots, Y_{a_{F+1}}\right\}, Y_{a_{i}}\right]=0 .
$$

Looking at the various brackets, one immediately observes that a Lie algebra of order $F$ is endowed with two different products: one binary given by the usual commutators, and one of order $F$ given by a fully symmetric product. Furthermore, a direct inspection of (2.1) and (2.2) shows that Lie algebras of order $F$ are $F$-ary extensions of Lie superalgebras, where the anticommutator is replaced by a fully symmetric bracket of order $F$. Many examples of Lie algebras of order $F$ were given in [9], and a formal study of this type of structure was initiated in [10, 11, 12.

Subsequently, a program of investigation of higher orders extensions of the Poincaré algebra, in the framework of Lie algebras of order $F$, was undertaken. Among various possibilities cubic and quartic extensions of the Poincaré algebra have been defined [9]. The cubic extension of the Poincaré algebra $I \mathfrak{s o}_{3}(1,3)=\mathfrak{g}_{0} \oplus \mathfrak{g}_{1}$, with $\mathfrak{g}_{0}=I \mathfrak{s o}(1,3)=\left\langle L_{\mu \nu}=-L_{\nu \mu}, P_{\mu}, 0 \leq \mu, \nu \leq 3\right\rangle$ generating the Poincaré algebra and $\mathfrak{g}_{1}=\left\langle V_{\mu}, 0 \leq \mu \leq 3\right\rangle$ being the vector representation, is defined by the brackets

$$
\begin{aligned}
{\left[L_{\mu \nu}, L_{\rho \sigma}\right] } & =\eta_{\nu \sigma} L_{\rho \mu}-\eta_{\mu \sigma} L_{\rho \nu}+\eta_{\nu \rho} L_{\mu \sigma}-\eta_{\mu \rho} L_{\nu \sigma} \\
{\left[L_{\mu \nu}, P_{\rho}\right] } & =\eta_{\nu \rho} P_{\mu}-\eta_{\mu \rho} P_{\nu},\left[L_{\mu \nu}, V_{\rho}\right]=\eta_{\nu \rho} V_{\mu}-\eta_{\mu \rho} V_{\nu},\left[P_{\mu}, V_{\nu}\right]=0 \\
\left\{V_{\mu}, V_{\nu}, V_{\rho}\right\} & =\eta_{\mu \nu} P_{\rho}+\eta_{\mu \rho} P_{\nu}+\eta_{\rho \nu} P_{\mu},
\end{aligned}
$$

where $\eta_{\mu \nu}=\operatorname{diag}(1,-1,-1,-1)$ is the Minkowski metric.

The quartic extensions of the Poincaré algebra are constructed by considering two Majorana spinors. In the $\mathfrak{s l}(2, \mathbb{C}) \cong \mathfrak{s o}(1,3)$ notations of dotted and undotted indices, a left handed spinor is given by $\psi_{L}{ }^{\alpha}$ and a a right-handed spinors by $\bar{\psi}_{R \dot{\alpha}}$. The spinor conventions to raise/lower indices are as follows $\psi_{L \alpha}=\varepsilon_{\alpha \beta} \psi_{L}{ }^{\beta}, \psi_{L}^{\alpha}=\varepsilon^{\alpha \beta} \psi_{L \beta}, \bar{\psi}_{R \dot{\alpha}}=\varepsilon_{\dot{\alpha} \dot{\beta}} \bar{\psi}_{R}{ }^{\dot{\beta}}, \bar{\psi}_{R}^{\dot{\alpha}}=\varepsilon^{\dot{\alpha} \dot{\beta}} \bar{\psi}_{R \dot{\beta}}$ with $\left(\psi_{\alpha}\right)^{*}=\bar{\psi}_{\dot{\alpha}}$, $\varepsilon_{12}=\varepsilon_{\dot{1} \dot{2}}=-1, \varepsilon^{12}=\varepsilon^{i \dot{2}}=1$. The $4 D$ Dirac matrices, in the Weyl representation, are

$$
\gamma_{\mu}=\left(\begin{array}{cc}
0 & \sigma_{\mu} \\
\bar{\sigma}_{\mu} & 0
\end{array}\right)
$$

with $\sigma_{\mu \alpha \dot{\alpha}}=\left(1, \sigma_{i}\right), \quad \bar{\sigma}_{\mu}^{\dot{\alpha} \alpha}=\left(1,-\sigma_{i}\right)$, where $\sigma_{i}, i=1,2,3$ are the Pauli matrices. With these notations, we introduce two series of Majorana spinors $Q^{I}{ }_{\alpha}, \bar{Q}_{I \dot{\alpha}}$ satisfying the relation $\left(Q^{I}{ }_{\alpha}\right)^{\dagger}=$ $\bar{Q}_{I \dot{\alpha}}$ and define the quartic extensions of the Poincaré algebra (we only give the quartic brackets) by 


$$
\begin{aligned}
\left\{Q^{I_{1}}{ }_{\alpha_{1}}, Q^{I_{2}}{ }_{\alpha_{2}}, Q^{I_{3}}{ }_{\alpha_{3}}, Q^{I_{4}} \alpha_{4}\right\} & =0 \\
\left\{Q^{I_{1}}{ }_{\alpha_{1}}, Q^{I_{2}}{ }_{\alpha_{2}}, Q^{I_{3}} \alpha_{3}, \bar{Q}_{I_{4} \dot{\alpha}_{4}}\right\} & =2 i\left(\delta^{I_{1}}{ }_{I_{4}} \varepsilon^{I_{2} I_{3}} \varepsilon_{\alpha_{2} \alpha_{3}} \sigma_{\alpha_{1} \dot{\alpha}_{4}}^{\mu}+\delta^{I_{2}} I_{4} \varepsilon^{I_{1} I_{3}} \varepsilon_{\alpha_{1} \alpha_{3}} \sigma_{\alpha_{2} \dot{\alpha}_{4}}^{\mu}\right. \\
& \left.+\delta^{I_{3}} I_{I_{4}} \varepsilon^{I_{1} I_{2}} \varepsilon_{\alpha_{1} \alpha_{2}} \sigma_{\alpha_{3} \dot{\alpha}_{4}}^{\mu}\right) P_{\mu} \\
\left\{Q^{I_{1} \alpha_{1}}, Q^{I_{2}}{ }_{\alpha_{2}}, \bar{Q}_{I_{3} \dot{\alpha}_{3}}, \bar{Q}_{I_{4} \dot{\alpha}_{4}}\right\} & =0,
\end{aligned}
$$

the remaining brackets involving three $\bar{Q}$ and one $Q$ or four $\bar{Q}$ are obtained by hermitian conjugation of the corresponding equation in (2.5) and $P_{\mu}^{\dagger}=-P_{\mu} 1$. In (2.5), $\varepsilon^{I J}$ is the $S U(2)$ invariant tensor given by $\varepsilon^{12}=-\varepsilon^{21}=1$. This tensor enables us to define $S U(2)$-invariant, or equivalently to raise or lower the indices. We define $Q_{I \alpha}=\varepsilon_{I J} Q^{J}{ }_{\alpha}$, with $\varepsilon_{I J} \varepsilon^{J K}=\delta_{I}{ }^{K}$.

The cubic extension (2.3) was intensively studied in the framework of Quantum Field Theory [13, 14, 22, 23. However, it has been proven in [14] that in four dimensional space-time, and for a specific representation of (2.3), no-interacting terms are allowed. In order to get more precise insight of the above higher order extensions, and to identify interesting representations of (2.3) or (2.5), one possible direction would be to construct an adapted superspace, where the algebra is realised upon differential operators.

\section{Superspace for higher order extensions of the Poincaré algebra}

In this section, we construct an adapted superspace leading to an ad hoc realisation of the algebras (2.3) and (2.5). More precisely, we introduce adapted variables such that the transformations generated by $V$ and $Q$ correspond to a translation in some appropriate "internal" space hereafter called superspace. We impose further that the algebra is realised by means of differential operators. The various variables and differential operators, together with the basic relations they have to satisfy, will be introduced progressively. It appears at the very end that the order two/three parafermions turn out to be the relevant variables [2]. Moreover, it is important to emphasise that the parafermionic variables appear quite naturally, but in a different way as they appeared historically in the literature [3, 4]. Since the construction is analogous for the cubic algebra (2.3) and for the quadratic algebra (2.5), in the next subsection we construct the (ternary) superspace associated to (2.3) with many details. Subsection 3.2 is devoted to the analogous construction in the quartic case. However, it will be shown that the latter case exhibits an exceptional behaviour, and a quaternary superspace can even be constructed with fermions.

\subsection{Ternary superspace}

A major progress towards the understanding of Lie algebras of order three was achieved when it was realised that groups associated to Lie algebras of order three may be defined [11. Indeed, it was a priori not obvious that groups associated to ternary algebras can be defined, since for a group the multiplication of two elements is always defined, although for a ternary algebra only the multiplication of three elements is defined. Moreover, the group structure enables us to identify the parameters of the transformation [11]. These parameters turn out to be the natural cubic generalisation of the Grassmann algebra (or the exterior algebra) called the three-exterior algebra.

\footnotetext{
${ }^{1}$ With our conventions since there is no $i$ factors in the commutators, we have that $P_{\mu}=\partial_{\mu}$, thus the physical quadri-momentum is given by $-i P_{\mu}$.
} 
This algebra is generated by four real variables $\theta^{\mu}$ which are in the vector representation of the Lorentz algebra and which satisfy the cubic relation

$$
\left\{\theta^{\mu}, \theta^{\nu}, \theta^{\rho}\right\}=\theta^{\mu} \theta^{\nu} \theta^{\rho}+\theta^{\nu} \theta^{\rho} \theta^{\mu}+\theta^{\rho} \theta^{\mu} \theta^{\nu}+\theta^{\mu} \theta^{\rho} \theta^{\nu}+\theta^{\nu} \theta^{\mu} \theta^{\rho}+\theta^{\rho} \theta^{\nu} \theta^{\mu}=0 .
$$

This algebra can be defined over the real or complex fields. For more details concerning the threeexterior algebra one can see $e . g$. [11. In the sequel we only consider the real three-exterior algebra. This algebra was introduced long time ago by N. Roby [18], and for that reason will call it from now on the Roby algebra. Since the transformation parameters belong to the Roby algebra, it is natural to postulate that the superspace is generated by

$$
X=\left(x^{\mu}, \theta^{\mu}\right),
$$

where $x^{\mu}$ belongs to the Minkowski space-time and $\theta^{\mu}$ are the generators of the Roby algebra. This identification is the natural cubic extension of the superspace considered in usual supersymmetric theories.

We further need to introduce the notion of "conjugate" momentum of the variables $X$. To this extent, we have to identify some derivative $\partial_{\mu}$ associated to the variables $\theta^{\mu}$. To identify the action of $\partial_{\mu}$ on the $\theta^{\nu}$, we assume further that a differential realisation of the Lorentz algebra can be constructed from the variables $\theta$ and their derivative $\partial$. We know that the variables $\theta$ are Lorentz vectors. Following Green [3, 4], the more general quantisation which ensures that $\theta^{\mu}$ are vectors of the Lorentz algebra is given by the parafermions 2 . We thus assume the parafermionic relation 3

$$
\begin{array}{ll}
{\left[\left[\theta^{\mu}, \theta^{\nu}\right], \theta^{\rho}\right]=0,} & {\left[\left[\theta^{\mu}, \theta^{\nu}\right], \partial_{\rho}\right]=-\delta^{\mu}{ }_{\rho} \theta^{\nu}+\delta^{\nu}{ }_{\rho} \theta^{\mu},} \\
{\left[\left[\theta^{\mu}, \partial_{\nu}\right], \theta^{\rho}\right]=\delta_{\nu}{ }^{\rho} \theta^{\mu},} & {\left[\left[\theta^{\mu}, \partial_{\nu}\right], \partial_{\rho}\right]=-\delta^{\mu}{ }_{\rho} \partial_{\nu},} \\
{\left[\left[\partial_{\mu}, \partial_{\nu}\right], \theta^{\rho}\right]=-\delta_{\mu}{ }^{\rho} \partial_{\nu}+\delta_{\nu}{ }^{\rho} \partial_{\mu}} & {\left[\left[\partial_{\mu}, \partial_{\nu}\right], \partial_{\rho}\right]=0 .}
\end{array}
$$

As a consequence, if we define

$$
\mathcal{J}_{\mu \nu}=\left[\theta_{\nu}, \partial_{\mu}\right]-\left[\theta_{\mu}, \partial_{\nu}\right]
$$

the relations (3.3) ensure that (3.4) act correctly on $\theta$ and $\partial$ :

$$
\left[\mathcal{J}_{\mu \nu}, \theta_{\rho}\right]=\eta_{\nu \rho} \theta_{\mu}-\eta_{\mu \rho} \theta_{\nu} .
$$

Introducing further $P_{\mu}$, the conjugate momentum of $x^{\nu}\left(\left[P_{\mu}, x^{\nu}\right]=\delta_{\mu}{ }^{\nu}\right)$, we thus define the Lorentz generators as

$$
L_{\mu \nu}=x_{\nu} P_{\mu}-x_{\mu} P_{\nu}+\mathcal{J}_{\mu \nu}
$$

Since we are considering ternary algebras involving fully symmetric products, putting (3.1) and (3.3) together shows explicitly that we are considering parafermions of order two. This means that the relations (3.1) have to be supplemented by [4]

$$
\begin{aligned}
& \left\{\theta^{\mu}, \theta^{\nu}, \theta^{\rho}\right\}=0, \\
& \left\{\theta^{\mu}, \theta^{\nu}, \partial_{\rho}\right\}=2 \delta^{\mu}{ }_{\rho} \theta^{\nu}+2 \delta^{\nu}{ }_{\rho} \theta^{\mu}, \\
& \left\{\theta^{\mu}, \partial_{\nu}, \partial_{\rho}\right\}=2 \delta^{\mu}{ }_{\nu} \partial_{\rho}+2 \delta^{\mu}{ }_{\rho} \partial_{\nu}, \\
& \left\{\partial_{\mu}, \partial_{\nu}, \partial_{\rho}\right\}=0 .
\end{aligned}
$$

\footnotetext{
${ }^{2}$ Or parabosons, but the parabosonic algebra is incompatible with requirement (3.1).

${ }^{3}$ It should be noted that not all the relations (3.3) are independent, and some are related through Jacobi identities.

${ }^{4}$ In the literature the brackets (3.3) and (3.7) are unified $<\theta^{\mu}, \partial_{\rho}, \theta^{\nu}>=\delta^{\mu}{ }_{\rho} \theta^{\nu}+\delta^{\nu}{ }_{\rho} \theta^{\mu},<\theta^{\mu}, \theta^{\nu}, \partial_{\rho}>=\delta^{\nu}{ }_{\rho} \theta^{\mu}$, where $\langle A, B, C\rangle=A B C+C B A$ etc.
} 
It is interesting to observe that the construction leading to (3.7) and (3.3) goes in reverse order to that of parafermionic algebras. Historically, parafermions were defined by means of equation (3.3), in order to realise the Lorentz algebra. After all the order of paraquantisation (here two, but in general $p$ ) are specified by assuming on which representation of the Lorentz algebra the parafermionic algebra acts. Order $p$ parafermionic algebras involved fully symmetric brackets of order $p+1$ and, in particular, order two parafermionic algebra give rise to the brackets (3.7). However, in our construction, the cubic brackets (3.1) are obtained from the very beginning, by our superspace assumption. Finally, notice that the order two parafermionic algebra (3.3), (3.7) is a non-faithful representation of the algebra (3.2) since with respect to the Roby algebra we have one more relation $\left[\left[\theta^{\mu}, \theta^{\nu}\right], \theta^{\rho}\right]=0$.

However, we cannot expect to construct a differential operator $V_{\mu}$ from $\partial_{\mu}$ and $\theta^{\mu}$ acting on $\theta^{\mu}$ and satisfying the cubic relations (2.3). Indeed, the relations (3.3) and (3.7) are cubic, meaning that no bilinear relations upon $\theta^{\mu}$ and $\partial_{\nu}$ are given and consequently no action of $\partial_{\mu}$ on $\theta^{\nu}$ is specified 6 . This situation is very similar to the implementation of the Noether theorem within the framework of ternary symmetries, where the conserved charges generate the symmetry through quadratic relations using the usual quantisation procedure (e.g. the equal-time (anti)-commutation relations) [13, 22, 23]. We have shown in [13, 22, that if we have an invariant Lagrangian $\mathcal{L}(\Phi)$ (where $\Phi$ is a given multiplet of the algebra (2.3) ) with conserved charges $\hat{L}_{\mu \nu}, \hat{P}_{\mu}, \hat{V}_{\mu}$ such that after quantisation we have the transformation laws $\left[\hat{L}_{\mu \nu}, \Phi\right],\left[\hat{P}_{\mu}, \Phi\right],\left[\hat{V}_{\mu}, \Phi\right]$, the algebra is realised through multiple-commutators

$$
\left[\hat{V}_{\mu},\left[\hat{V}_{\nu},\left[\hat{V}_{\rho}, \Phi\right]\right]\right]+\text { perm. }=\eta_{\mu \nu}\left[\hat{P}_{\rho}, \Phi\right]+\eta_{\nu \rho}\left[\hat{P}_{\mu}, \Phi\right]+\eta_{\mu \rho}\left[\hat{P}_{\nu}, \Phi\right] .
$$

This procedure is standard in the implementation of Lie (super)algebra in Quantum Field Theory, but the equation corresponding to (3.8) in this case is not the end of the story since the Jacobi identities allow to obtain a relation which is independent of the fields $\Phi$. But here, in the context of ternary symmetries, the situation is very different, since the Jacobi identities (2.2) do not allow to write the algebra in a $\Phi$ independent form. This weaker realisation of the algebra has the interesting consequence that it enables us to consider algebraic structure (in Quantum Field Theories), different from Lie superalgebras, without contradicting the spin-statistics theorem (see [23] for a discussion). Finally, it is a matter of calculation to check that the Jacobi identities are satisfied by the realisation (3.8).

As we have recalled briefly, the implementation of the Noether theorem in higher order algebras automatically leads to an algebraic realisation through multiple commutators. Since for the parafermionic algebra, the natural action is defined also by means of commutators (see (3.3)), it is tempting to try to define a superspace in which the algebra is realised in the form of (3.8). Then, the parafermionic algebra will be the cornerstone of the realisation of the algebra (2.3) on the superspace $X=\left(x^{\mu}, \theta^{\mu}\right)$. We then introduce the parameters of the transformations $\varepsilon^{\mu}$ such that we have the transformation

$$
\theta^{\mu} \rightarrow \theta^{\prime \mu}=\theta^{\mu}+\varepsilon^{\mu}
$$

under (2.3). This means that the variables $\theta^{\prime}$ are of the same type as the variables $\theta$. So do the variables $\varepsilon$. Now we would like to define the generators of our algebra. The algebra (2.3) is cubic

\footnotetext{
${ }^{5}$ In particular the Roby algebra is infinitely many generated [18, 11] although the order three parafermionic algebra is finite dimensional (see Section 4).

${ }^{6}$ It is possible, however, to obtain matrix representations of (2.3) by postulating cubic relations analogous to (3.7) of 13 .
} 
and the variables $\varepsilon$ satisfy also the cubic relations (3.3) and (3.7). It is known that in general the tensor product of two algebras has no meaning (and this is even a more difficult task for algebras defined by cubic relations like (2.3) and (3.7), (3.3)). We thus assume that the variables $\varepsilon$ and the generators of the transformation are indissociable. Since for the para-Grassmann algebra the natural objects are the commutators, it is natural to define $V$ by means of a commutator. There are two parts in the generators $V$. One leading to the transformations on the variables $\theta$ and one transforming the variables $x: V=V_{\theta}+V_{x}$ such that $\left[V_{\theta}, x\right]=0$ and $\left[V_{x}, \theta\right]=0$. Having only the variables $\theta^{\mu}$ and the parameters $\varepsilon^{\mu}$ it is not difficult to observe that it is not possible to define a $V_{x}$ commuting with the $\theta$ 's and being a Lorentz scalar. We thus introduce one more parameter $\theta$ which is a paragrassmann variable in the scalar representation of the Lorentz algebra ${ }^{7}$ such that

$$
V=\left[\varepsilon^{\mu}, \partial_{\mu}\right]+\left[\theta, \theta^{\mu}\right]\left[\varepsilon^{\sigma}, \theta_{\mu}\right] P_{\sigma},
$$

which gives

$$
\delta \theta^{\alpha}=\left[V, \theta^{\alpha}\right]=\varepsilon^{\alpha}, \quad \delta x^{\alpha}=\left[V, x^{\alpha}\right]=\left[\theta, \theta^{\mu}\right]\left[\varepsilon^{\alpha}, \theta_{\mu}\right] .
$$

It is important to realize that, the $\delta x^{\alpha}$ are commuting real variables.

Having constructed a differential realisation of the cubic extension of the Poincaré algebra (2.3), we are now looking for the closure of the algebra. We have to check the algebra in the sense of (3.8) on any monomial in $\theta$. In particular, we have

$$
\begin{aligned}
{\left[V_{1},\left[V_{2},\left[V_{3}, \theta^{\alpha_{1}} \theta^{\alpha_{2}} \theta^{\alpha_{3}}\right]\right]\right] } & =\varepsilon_{1}^{\alpha_{1}} \varepsilon_{2}^{\alpha_{2}} \varepsilon_{3}^{\alpha_{3}}+\varepsilon_{2}^{\alpha_{1}} \varepsilon_{3}^{\alpha_{2}} \varepsilon_{1}^{\alpha_{3}}+\varepsilon_{3}^{\alpha_{1}} \varepsilon_{1}^{\alpha_{2}} \varepsilon_{2}^{\alpha_{3}} \\
& +\varepsilon_{1}^{\alpha_{1}} \varepsilon_{3}^{\alpha_{2}} \varepsilon_{2}^{\alpha_{3}}+\varepsilon_{2}^{\alpha_{1}} \varepsilon_{1}^{\alpha_{2}} \varepsilon_{3}^{\alpha_{3}}+\varepsilon_{3}^{\alpha_{1}} \varepsilon_{2}^{\alpha_{2}} \varepsilon_{1}^{\alpha_{3}}
\end{aligned}
$$

which is fully symmetric in the indices $1,2,3$. This means that $\left[V_{1},\left[V_{2},\left[V_{3}, \theta^{\alpha_{1}} \theta^{\alpha_{2}} \theta^{\alpha_{3}}\right]+\right.\right.$ perm, never vanishes (in order to simplify the notations, we denote $V_{1} \cdot V_{2} \cdot V_{3} \cdot \theta=\left[V_{1},\left[V_{2},\left[V_{2}, \theta\right]\right]\right]$ and $\left\{V_{1}, V_{2}, V_{3}\right\} . \theta=\left[V_{1},\left[V_{2},\left[V_{2}, \theta\right]\right]\right]+$ perm. etc.). It turns out that this problem is independent of the realisation of the operator $V$. Actually, if we simply assume to have an operator $\delta$ such that $\delta . \theta=\varepsilon$, together with the Leibniz rule, we automatically have for $\delta_{1} \delta_{2} \delta_{3}\left(\theta^{\alpha_{1}} \theta^{\alpha_{2}} \theta^{\alpha_{3}}\right)$ the R.H.S. of (3.11). In fact this discrepancy is already present in supersymmetric theories. But in this case, since the parameters are anticommuting Grassmann parameters, the natural solution is to replace anticommutators by commutators when the parameters are taken into account. In our case we have some analogy to the procedure above. Recall that the formal study of Lie algebras of order three [11] leads naturally to a $\mathbb{Z}_{3}$-twisted tensor product. This means, in particular, that if we consider three successive transformations $\varepsilon_{1}, \varepsilon_{2}, \varepsilon_{3}$, we have a $\mathbb{Z}_{3} \times \mathbb{Z}_{3} \times \mathbb{Z}_{3}$ graded structure. As a consequence, the algebraic structure which emerges from this grading is a ternary analog of the colour algebras were the bracket is no more fully symmetric [24] 8 . This means that, as in the case of usual Lie algebras, the introduction of the parameters of the transformation forces us to consider a different, but related algebraic structure. This can be seen as some analogy to the Jordan-Wigner transformations in that context. This is the inverse process of the decoloration theorem proved in [12, which states that a ternary colour algebra is isomorphic to a Lie algebra of order three. Ternary colour algebras have been studied in [12. The basic tool to define colour algebras is a

\footnotetext{
${ }^{7}$ This additional variable can be understood as coming from a compactification of the $(1+4) D$ to the $(1+3) D$ Minkowski space-time. Thus $\left(\theta^{\mu}, \theta\right)$ is in the vector representation of $S O(1,4)$.

${ }^{8}$ Colour algebras were introduced as a possible generalisation of Lie (super)algebras, were the brackets are neither symmetric nor antisymmetric. In the various brackets the usual plus or minus sign of the (anti)commutators is substituted by a commutation factor.
} 
grading determined by an Abelian group. Here the grading group is given by $\mathbb{Z}_{3} \times \mathbb{Z}_{3} \times \mathbb{Z}_{3}$. The latter, besides defining the underlying grading in the structure, moreover provides a new object known as commutation factor defined by

$$
N(\vec{a}, \vec{b})=q^{a_{1}\left(b_{2}+b_{3}\right)+a_{2} b_{3}-b_{1}\left(a_{2}+a_{3}\right)-b_{2} a_{3}},
$$

where $q=e^{\frac{2 i \pi}{3}}$ and $\vec{a}, \vec{b} \in \mathbb{Z}_{3}^{3}$. The trilinear bracket is now defined by 9

$$
\begin{aligned}
\left\{\left|V_{1}, V_{2}, V_{3}\right|\right\}_{N} & =V_{1} V_{2} V_{3}+N\left(\operatorname{gr}\left(\varepsilon_{1}\right), \operatorname{gr}\left(\varepsilon_{2}\right)+\operatorname{gr}\left(\varepsilon_{3}\right)\right) V_{2} V_{3} V_{1} \\
& +N\left(\operatorname{gr}\left(\varepsilon_{1}\right)+\operatorname{gr}\left(\varepsilon_{2}\right), \operatorname{gr}\left(\varepsilon_{3}\right)\right) V_{3} V_{1} V_{2} \\
& +N\left(\operatorname{gr}\left(\varepsilon_{2}\right), \operatorname{gr}\left(\varepsilon_{3}\right)\right) V_{1} V_{3} V_{2}+N\left(\operatorname{gr}\left(\varepsilon_{1}\right), \operatorname{gr}\left(\varepsilon_{2}\right)\right) V_{2} V_{1} V_{3} \\
& +N\left(\operatorname{gr}\left(\varepsilon_{1}\right), \operatorname{gr}\left(\varepsilon_{2}\right)\right) N\left(\operatorname{gr}\left(\varepsilon_{1}\right), \operatorname{gr}\left(\varepsilon_{3}\right)\right) N\left(\operatorname{gr}\left(\varepsilon_{2}\right), \operatorname{gr}\left(\varepsilon_{3}\right)\right) V_{3} V_{2} V_{1} .
\end{aligned}
$$

In our case, with the commutation factor given by (3.12), and $\operatorname{gr}\left(\varepsilon_{1}\right)=(1,0,0), \operatorname{gr}\left(\varepsilon_{2}\right)=$ $(0,1,0), \operatorname{gr}\left(\varepsilon_{3}\right)=(0,0,1)$, the cubic brackets adopt the following form

$$
\left\{\left|V_{1}, V_{2}, V_{3}\right|\right\}_{N}=V_{1} V_{2} V_{3}+q^{2} V_{2} V_{3} V_{1}+q^{2} V_{3} V_{1} V_{2}+q V_{1} V_{3} V_{2}+q V_{2} V_{1} V_{3}+V_{3} V_{2} V_{1}
$$

In particular, since the constraint $1+q+q^{2}=0$ is satisfied and $V_{1} \cdot V_{2} \cdot V_{3} \cdot\left(\theta^{\alpha_{1}} \cdots \theta^{\alpha_{n}}\right)$ is fully symmetric in the subindices $1,2,3$, we automatically have that

$$
\left\{\left|V_{1}, V_{2}, V_{3}\right|\right\}_{N} \cdot\left(\theta^{\alpha_{1}} \cdots \theta^{\alpha_{n}}\right)=0 .
$$

Performing a similar computation for the space-time coordinates, we obtain the identities

$$
\begin{aligned}
\left\{\left|V_{1}, V_{2}, V_{3}\right|\right\}_{N} \cdot x^{\alpha}= & -q^{2}\left[\theta, \varepsilon_{2}^{\mu}\right]\left[\varepsilon_{3}^{\alpha}, \varepsilon_{1 \mu}\right]-q^{2}\left[\theta, \varepsilon_{1}^{\mu}\right]\left[\varepsilon_{3}^{\alpha}, \varepsilon_{2 \mu}\right] \\
& -\left[\theta, \varepsilon_{2}^{\mu}\right]\left[\varepsilon_{1}^{\alpha}, \varepsilon_{3 \mu}\right]-\left[\theta, \varepsilon_{3}^{\mu}\right]\left[\varepsilon_{1}^{\alpha}, \varepsilon_{2 \mu}\right] \\
& -q\left[\theta, \varepsilon_{1}^{\mu}\right]\left[\varepsilon_{2}^{\alpha}, \varepsilon_{3 \mu}\right]-q\left[\theta, \varepsilon_{3}^{\mu}\right]\left[\varepsilon_{2}^{\alpha}, \varepsilon_{1 \mu}\right]=a^{\alpha} .
\end{aligned}
$$

It is important to notice that the $a^{\beta}$ are complex numbers. This means that the "coloration" of the algebra $I \mathfrak{s o}_{3}(1.3)$, coming from our adapted Jordan-Wigner transformation gives rise to the algebra (3.14), which is manifestly a complex algebra since the structure constants are complex 10. This deserves some explanation. The $\varepsilon$ are real parafermions, therefore the transformation properties (3.10) ensure that $\delta x$ and $\delta \theta$ are both real. However, since $a^{\beta}$ is complex, this means that the cubic algebra (2.3) is realised in a complexification of the superspace $(x, \theta)$. In other words, the algebra cannot be realised on a real vector space. This is the best possible result in this direction using this ansatz.

\footnotetext{
${ }^{9}$ There is also some additional Jacobi identities which are however not relevant for our analysis.

${ }^{10}$ In fact since $\mathbb{Z}_{3}^{3}$ is complex, the grading makes sense only in a complexification $I \mathfrak{s o} \mathfrak{o}_{3}(1.3) \otimes_{\mathbb{R}} \mathbb{C}$ of the cubic algebra $I \mathfrak{s o}_{3}(1.3)$.
} 


\subsection{Quaternary superspace}

The construction of quaternary superspaces goes along the same lines of the construction of cubic superspace, but with some differences that we give now. Firstly the internal variables are given by order three parafermions instead of order two parafermions. Furthermore these variables are in the spinor representations of the Lorentz algebra (we have two Majorana spinors): $\theta^{I}{ }_{\alpha}, \bar{\theta}_{I \dot{\alpha}}$ with $\theta^{I}{ }_{\alpha}^{\dagger}=\bar{\theta}_{I \dot{\alpha}}$. Denoting generically by $\theta^{a}$ and $\partial_{a}$ the order three parafermions and their associated momenta, the order three parafermionic algebra is then given by (3.3) and the quartic relations

$$
\begin{aligned}
\left\{\theta^{a_{1}}, \theta^{a_{2}}, \theta^{a_{3}}, \theta^{a_{4}}\right\} & =0 \\
\left\{\theta^{a_{1}}, \theta^{a_{2}}, \theta^{a_{3}}, \partial_{a_{4}}\right\} & =5 \delta^{a_{1}}{ }_{a_{4}}\left\{\theta^{a_{2}}, \theta^{a_{3}}\right\}+5 \delta^{a_{2}}{ }_{a_{4}}\left\{\theta^{a_{1}}, \theta^{a_{3}}\right\}+5 \delta^{a_{3}}{ }_{a_{4}}\left\{\theta^{a_{1}}, \theta^{a_{2}}\right\}, \\
\left\{\theta^{a_{1}}, \theta^{a_{2}}, \partial_{a_{3}}, \partial_{a_{4}}\right\} & =5 \delta^{a_{1}}{ }_{a_{3}}\left\{\theta^{a_{2}}, \partial_{a_{4}}\right\}+5 \delta^{a_{1}}{ }_{a_{4}}\left\{\theta^{a_{2}}, \partial_{a_{3}}\right\}+5 \delta^{a_{2}} a_{3}\left\{\theta^{a_{1}}, \partial_{a_{4}}\right\} \\
& +5 \delta^{a_{2}}{ }_{a_{4}}\left\{\theta^{a_{1}}, \partial_{a_{3}}\right\}-\frac{9}{2} \delta^{a_{1}}{ }_{a_{3}} \delta^{a_{2}}{ }_{a_{4}}-\frac{9}{2} \delta^{a_{1}}{ }_{a_{4}} \delta^{a_{2}}{ }_{a_{3}},
\end{aligned}
$$

plus similar relations involving one $\theta$ and three $\partial$ or four $\partial$.

Introducing the parameters of the transformation, the quartic supercharges are now given by

$$
\begin{aligned}
& Q=\quad\left[\varepsilon^{I \alpha}, \partial_{I \alpha}\right]+2 i\left[\varepsilon^{I \alpha}, \theta_{I \alpha}\right]\left[\theta^{J \beta}, \bar{\theta}_{J}{ }^{\dot{\beta}}\right] \sigma_{\beta \dot{\beta}}^{\mu} P_{\mu}, \\
& \bar{Q}=-\quad\left[\bar{\partial}_{\dot{\alpha}}^{I}, \bar{\varepsilon}_{I}{ }^{\dot{\alpha}}\right]-2 i\left[\theta^{J \beta}, \bar{\theta}_{J} \dot{\beta}^{\dot{\beta}}\right]\left[\bar{\theta}_{\dot{\alpha}}^{I}, \bar{\varepsilon}_{I}{ }^{\dot{\alpha}}\right] \sigma_{\beta \dot{\beta}}^{\mu} P_{\mu} .
\end{aligned}
$$

leading to the transformations

$$
\begin{gathered}
\delta x^{\mu}=\left[Q, x^{\mu}\right]+\left[\bar{Q}, x^{\mu}\right]=2 i\left(\left[\varepsilon^{I \alpha}, \theta_{I \alpha}\right]\left[\theta^{J \beta}, \bar{\theta}_{J} \dot{\beta}^{\prime}\right]-\left[\theta^{J \beta}, \bar{\theta}_{J} \dot{\beta}^{\dot{\beta}}\right]\left[\bar{\theta}_{\dot{\alpha}}, \bar{\varepsilon}_{I} \dot{\alpha}^{\dot{\alpha}}\right]\right) \sigma_{\beta \dot{\beta}}^{\mu}, \\
\delta \theta^{I}{ }_{\alpha}=\left[Q, \theta_{\alpha}^{I}\right]=\varepsilon_{\alpha}^{I}, \quad \delta \bar{\theta}_{I \dot{\alpha}}=\left[\bar{Q}, \bar{\theta}_{I \dot{\alpha}}\right]=\bar{\varepsilon}_{I \dot{\alpha}} .
\end{gathered}
$$

A direct inspection shows that $\delta x^{\mu}$ is real.

Finally, as in the cubic case, the algebra is realised in a complexification of the quaternary superspace, but now with a $\mathbb{Z}_{4} \times \mathbb{Z}_{4} \times \mathbb{Z}_{4} \times \mathbb{Z}_{4}$-grading, where the corresponding commutation factor is given by

$$
N(\vec{a}, \vec{b})=i^{a_{1}\left(b_{2}+b_{3}+b_{4}\right)+a_{2}\left(b_{3}+b_{4}\right)+a_{3} b_{4}-b_{1}\left(a_{2}+a_{3}+a_{4}\right)-b_{2}\left(a_{3}+a_{4}\right)-b_{3} a_{4}} .
$$

We consider then four successive transformations $Q_{1}, \cdots, Q_{4}$ with grading $\operatorname{gr}\left(\varepsilon_{1}\right)=\operatorname{gr}\left(\bar{\varepsilon}_{1}\right)=$ $(1,0,0,0), \operatorname{gr}\left(\varepsilon_{2}\right)=\operatorname{gr}\left(\bar{\varepsilon}_{2}\right)=(0,1,0,0), \operatorname{gr}\left(\varepsilon_{3}\right)=\operatorname{gr}\left(\bar{\varepsilon}_{3}\right)=(0,0,1,0), \operatorname{gr}\left(\varepsilon_{4}\right)=\operatorname{gr}\left(\bar{\varepsilon}_{4}\right)=(0,0,0,1)$ and define

$$
\begin{aligned}
& \left\{\left|Q_{1}, Q_{2}, Q_{3}, Q_{4}\right|\right\}_{N}=Q_{1}\left\{\left|Q_{2}, Q_{3}, Q_{4}\right|\right\}_{N}+N\left(\operatorname{gr}\left(\varepsilon_{1}\right), \operatorname{gr}\left(\varepsilon_{2}\right)\right) Q_{2}\left\{\left|Q_{1}, Q_{3}, Q_{4}\right|\right\}_{N} \\
& N\left(\operatorname{gr}\left(\varepsilon_{1}\right)+\operatorname{gr}\left(\varepsilon_{2}\right), \operatorname{gr}\left(\varepsilon_{3}\right)\right) Q_{3}\left\{\left|Q_{1}, Q_{2}, Q_{4}\right|\right\}_{N}+N\left(\operatorname{gr}\left(\varepsilon_{1}\right)+\operatorname{gr}\left(\varepsilon_{2}\right)+\operatorname{gr}\left(\varepsilon_{3}\right), \operatorname{gr}\left(\varepsilon_{4}\right)\right) Q_{4}\left\{\left|Q_{1}, Q_{2}, Q_{3}\right|\right\}_{N} \\
& =Q_{1}\left\{\left|Q_{2}, Q_{3}, Q_{4}\right|\right\}_{N}+i Q_{2}\left\{\left|Q_{1}, Q_{3}, Q_{4}\right|\right\}_{N}-Q_{3}\left\{\left|Q_{1}, Q_{2}, Q_{4}\right|\right\}_{N}-i Q_{4}\left\{\left|Q_{1}, Q_{2}, Q_{3}\right|\right\}_{N},
\end{aligned}
$$

with $\left\{\left|Q_{i}, Q_{j}, Q_{k}\right|\right\}_{N}$ given by (3.13), but with commutator factor (3.19) instead of (3.12). As in section 3.1 , we can easily check that $\left\{\left|Q_{1}, Q_{2}, Q_{3}, Q_{4}\right|\right\}_{N}$ vanishes on any polynomial on $\theta$ and generate a space-time translation with a complex parameter. 


\subsection{Fermions for higher order superspaces}

In principle, the variables $\theta$ should satisfy the Roby algebra $\{\theta, \theta, \theta\}=0$ for cubic extensions and the Roby algebra $\{\theta, \theta, \theta, \theta\}=0$ for quartic extensions, with no more relations among the $\theta$ 's but $[[\theta, \theta], \theta]=0$. However, if we "relax" these assumptions, this gives rise to the possibility to realise higher order algebras using only fermions. This realisation can be put on the same footing with the realisation of the algebra in relation with the Noether theorem, where the algebra is realised by means of bosons and fermions. The only relevant relations in the construction of ternary (resp. quaternary) superspaces are equations (3.3) and $\{\theta, \theta, \theta\}=0$ (resp. $\{\theta, \theta, \theta, \theta\}=0$ ). It turns out that usual fermions do satisfy (3.3) and (3.7) or (3.16) (but with a different normalisation for the last equations). This leads to an alternative construction of higher order superspaces, with usual fermions instead of parafermions. Of course that in the cubic case, this possibility is excluded since the generators $V_{\mu}$ are in the vector representation of the Lorentz algebra. Indeed, in this case superfields (see Section 4) will automatically generate commuting fermions or anticommuting bosons. However, for extensions involving spinors this possibility is still open. If one considers the quartic algebra (2.5), it is straightforward to verify that it admits generalisation in any space-time dimensions. As happens for supersymmetric theories, these extensions depend on the space-time dimension and of the properties of spinors. As we now show, there is one space-time dimension where these quartic algebras present some exceptional features, namely $D=10$.

Before giving the analog of (2.5) in ten space-time dimensions, lets us recall some properties of spinors which are useful for us. (For more details one can see e.g. [30].) Dirac spinors can be defined in any space-time dimensions and Weyl spinors in even space-time dimensions. A Majorana (or pseudo-Majorana) spinor, that is a real spinor, can be defined in $D=4,8,9,11$ (mod. 8) and a Majorana Weyl spinor is only defined in $D=10$ (mod. 8). In this brief section, we are mainly interested in the minimal quartic extensions, that is, when real spinors (or real Weyl spinors) do exist, thus when $D=9,10$ or $D=11$. Furthermore, since our extensions involve fully symmetric product we consider the case where the charge conjugation matrix is symmetric. If $\Gamma^{M}$ are the $\gamma$-matrices in $D$-dimensions and $C$ the charge-conjugation matrix defined by

$$
\left(\Gamma^{M}\right)^{t}= \pm C \Gamma^{M} C^{-1}
$$

where $M^{t}$ denotes the transpose of the matrix $M$. Both signs are possible when $D$ is even and the sign of the R.H.S. is fixed when $D$ is odd. For $D=9$, the matrices $C$ and $\Gamma^{M} C$ are both symmetric ( $C$ is denoted $C_{+}$) although in $D=10$, there is one choice of $C$ (denoted $C_{+}$), that ensures that $C_{+}$and $\Gamma^{M} C_{+}$are both symmetric. The other choice of $C$, (denoted $C_{-}$), implies that we have $C_{-}$antisymmetric and $\Gamma^{M} C_{-}$symmetric. Thus when $D=9,10$ Majorana (Weyl) spinors exist together with a symmetric charge conjugation matrix.

Now we are ready to give the quartic extension of the Poincaré algebra. As mentioned previously these extensions exist in any space-time dimensions, but due to the special properties of Diracmatrices in $D=9,10$, these extensions are simpler in these cases. Since they present some analogies, they will be presented on the same footing. Consider $Q_{A}$ a Majorana spinor and $\Gamma^{M}, M=0, \cdots, 8$ (resp. $M=0, \cdots, 9)$ the Dirac $\Gamma$-matrices in $D=9$ (resp. $D=10) 11$. The quartic extension analogous to (2.5) is given by

\footnotetext{
${ }^{11}$ In $D=10$ the charge conjugation matrices $C_{+}$or $C_{-}$connect left-handed to right-handed spinors. This means that we cannot consider a quartic extension of the Poincaré algebra with only a Weyl spinor. This can be seen in an equivalent way. Indeed, if $\psi_{L}$ denotes a left-handed spinor, we have that $\psi_{L} \otimes \psi_{L}=[1] \oplus[3] \oplus[5]_{+}$with $[p]$ a $p-$ form and $[5]+$ a self-dual five-form.
} 


$$
\begin{aligned}
\left\{Q_{A_{1}}, Q_{A_{2}}, Q_{A_{3}}, Q_{A_{4}}\right\} & =C_{+A_{1} A_{2}}\left(\Gamma^{M} C_{+}\right)_{A_{3} A_{4}} P_{M}+C_{+A_{1} A_{3}}\left(\Gamma^{M} C_{+}\right)_{A_{2} A_{4}} P_{M} \\
& +C_{+A_{1} A_{4}}\left(\Gamma^{M} C_{+}\right)_{A_{2} A_{3}} P_{M}+C_{+A_{2} A_{3}}\left(\Gamma^{M} C_{+}\right)_{A_{1} A_{4}} P_{M} \\
& +C_{+A_{2} A_{4}}\left(\Gamma^{M} C_{+}\right)_{A_{1} A_{3}} P_{M}+C_{+A_{3} A_{4}}\left(\Gamma^{M} C_{+}\right)_{A_{1} A_{2}} P_{M} .
\end{aligned}
$$

Among these two extensions, the $D=10$ quartic extension of the Poincare algebra is very special. There exist two charge conjugation matrices $C_{+}$and $C_{-}$with different properties of symmetry. As in the preceding subsection, one can define a quaternary supercharge, considering order three parafermions in the Majorana representation of $S O(1,9)$. If we denote by $\theta^{A}, \partial_{A}$ the order three parafermions and by $\epsilon^{A}$ the parameters of the transformation, we have that

$$
Q=\left[\epsilon^{A}, \partial_{A}\right]+C_{-B C}\left[\theta^{B}, \theta^{C}\right]\left(\Gamma C_{+}\right)_{A D}\left[\epsilon^{A}, \theta^{D}\right]
$$

However, in this case, one is also able to construct a quaternary supercharge with usual fermions Introducing $\psi^{A}, \partial_{A}$ real Grassmann variables (in the Majorana representation of $S O(1,9)$ ) and their associated derivative we can construct in $D=10$ the quaternary supercharges using only fermions

$$
Q_{A}=\partial_{A}+\left(C_{-B C} \psi^{B} \psi^{C}\right)\left(\Gamma C_{+}\right)_{A D} \psi^{D}
$$

Of course, as happened in Section [3.2, the algebra is realised with multiple commutators in a complexification of the superspace. It is important to point out that this realisation is possible due to the special properties of the $C_{ \pm}$-matrices in ten space-time dimensions. Finally, looking to the algebraic structure (3.22) and its differential realisation (3.24) one may wonder whether this quartic relation is related with type IIA supersymmetry [26]?

To conclude this section let us mention that similar (complex) variables were used in [25] in a different context. In addition, order two paragrassmann algebras were used for the description of a superspace associated to parasupersymmetric extensions of the Poincaré algebra in [20].

\section{Superfields}

In the previous section we were able to construct an adapted superspace, where the underlying algebra (2.3) and (2.5) are realised in a differential way. Considering functions of the variables (3.2) (and its quartic analogue) gives us the opportunity to obtain various representations of the algebra (2.3) or (2.5). In analogy with supersymmetric theories, these functions will be called superfields. Since the definition of superfields is analogous in the ternary and quaternary cases, we study the first case with many details, the second being obtained in straightforward manner. In addition, we will analyze the extremely important problem of the existence of covariant derivatives. Although some serious obstruction exist in the full general case, slight modifications will enable us to find operators $D$ acting like covariant derivatives, in both the ternary and quartic cases. However, it turns out that we obtain, with this modification, an interesting effect, namely, space-time translations of null vectors. 


\subsection{Ternary superfields}

In what follows, we define a scalar superfield, i.e., a superfield invariant under Lorentz transformations. We then identify the various representations of the Lorentz algebra together with its spin content, which appears in the decomposition of the scalar superfield. We also analyse the possibility to construct another type of superfields using the so-called Green ansatz.

A scalar superfield is given by a function

$$
\Phi(x, \theta)
$$

Developing (4.1) with respect to the $\theta$, we get monomials in the parafermionic variables $\theta$. The first of the relations in (3.3) and (3.7), respectively, imply the identity

$$
\theta^{\mu} \theta^{\nu} \theta^{\rho}+\theta^{\rho} \theta^{\nu} \theta^{\mu}=0 .
$$

Upon successive application of the identity (4.2), we get the relation $\left(\theta^{\mu} \theta^{\nu} \theta^{\rho}\right)^{3}=0$. It can be shown that the relations (4.2) ensure that we have a finite number of monomials in the development of the superfield $\Phi$. To develop equation (4.1), we have to identify its components with respect to the Lorentz group. A series of results upon parafermions, that we recall now, has been established. Given an arbitrary monomial of degree $n$, say $\theta^{\alpha_{1}} \cdots \theta^{\alpha_{n}}$, it can be written as a linear combination of terms of the shape (see [4, 28, for details on the method)

$$
\begin{gathered}
{\left[\theta^{\mu_{1}}, \theta^{\nu_{1}}\right] \cdots\left[\theta^{\mu_{p}}, \theta^{\nu_{p}}\right]\left\{\theta^{\rho_{1}}, \cdots, \theta^{\rho_{q}}\right\},} \\
2 p+q=n, 0 \leq q \leq 2,0 \leq \alpha^{\mu_{1}}, \cdots, \alpha^{\mu_{p}}, \alpha^{\nu_{1}}, \cdots, \alpha^{\nu_{p}}, \alpha^{\rho_{1}}, \cdots, \alpha^{\rho_{q}} \leq 3 .
\end{gathered}
$$

The second result is even more interesting for our approach. It is known that an arbitrary tensor of order $n$ decomposes into irreducible representations of the Lorentz group characterised by a certain Young tableau [27. Moreover, multiplicity of representations in the above decomposition is generally greater than one. However, for order two parafermions (3.3), (3.7) the situation changes drastically. If we define $\mathcal{P}_{n}=\left\{\theta^{\alpha_{1}} \cdots \theta^{\alpha_{n}}, 0 \leq \alpha_{1}, \cdots, \alpha_{n} \leq 3\right\}$, it turns out that $\mathcal{P}_{n}$ contains one and only one irreducible subspace corresponding to each possible Young diagram of $n$ squares whose first row consists of not more than 2 squares $[4,29] 12$. This property of multiplicity of equivalent representations is one of the most appealing characteristics of parafermions. Technically, since to each allowed Young diagram there corresponds only one irreducible representation, we are able to chose the Young tableaux which gives us the more convenient results.

We will not give a systematic study of the decomposition of the superfield $\Phi$ upon the various Young tableaux, since these computations are straightforward but lengthy. We only give the general method to identify the spin content of the fields appearing in the decomposition of the superfield $\Phi$. We first recall that, in order to identify a representation associated to a given Young tableau, one as to define the Young symmetriser associated to the considered Young tableau. It is written as $P=S A$, i.e., we first antisymmetrise the columns and then symmetrise the rows. As an illustration, we give one example. Consider the representation associated to the Young tableau 13

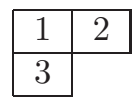

\footnotetext{
${ }^{12}$ Analogous properties are equally valid for order $p$ parafermions.

${ }^{13}$ If along the same lines, one calculates the representation associated to the Young tableau \begin{tabular}{|l|l|l|l|}
\hline 1 & 3 \\
\cline { 2 - 4 } one obtains
\end{tabular} $\frac{1}{3}\left[\theta^{M_{3}},\left[\theta^{M_{1}}, \theta^{M_{2}}\right]\right]$, which vanishes.
} 
its Young symmetriser is given by $P=\frac{1}{3}(1+(12))(1-(13))$ (where $(a b)$ means the transposition of $a$ and $b)$ and correspondingly it leads to the representation 14

$$
P \theta^{M_{1}} \theta^{M_{2}} \theta^{M_{3}}=\frac{1}{3}\left\{\theta^{M_{1}}, \theta^{M_{2}}\right\} \theta^{M_{3}}-\frac{1}{3}\left\{\theta^{M_{2}}, \theta^{M_{3}}\right\} \theta^{M_{1}} .
$$

Proceeding along the same lines, we obtain 17 representations, varying from the trivial representation to the one-dimensional representation specified to the Young tableau

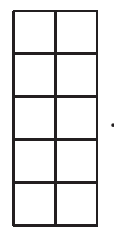

The tensors appearing in this decomposition can be seen to constitute irreducible representations of $G L(1,4)$, thus to identify representations of $S O(1,3)$, we first have to identify its $G L(1,3)$ content and then we have to extract traceless tensors. For instance, the $G L(1,4)$-tensor $\left\{\theta^{M}, \theta^{N}\right\}$ gives rise to the $G L(1,3)$ tensors $\left\{\theta^{\mu}, \theta^{\nu}\right\}$ and $\left\{\theta^{\mu}, \theta\right\}$. The first one leads then to $\theta^{\mu} \theta_{\mu}$ and $\left\{\theta^{\alpha}, \theta^{\beta}\right\}-\frac{1}{2} \theta^{\mu} \theta_{\mu} \eta^{\alpha \beta}$. Having identified the $S O(1,3)$ content of the decomposition of the various fields, we can now identify the spin or the helicity content of the various representations. For the former identification, corresponding to massive particles, we have to study the embedding $\mathfrak{s o}(3) \subset \mathfrak{s o}(1,3)$, although for massless particles, we have to decompose the various tensors according to the reduction chain $\mathfrak{s o}(2) \subset \mathfrak{s o}(1,3)$. Considering only non-isomorphic representations of $\mathfrak{s o}(1,3)$, we obtain for the massive case

$$
\begin{aligned}
& \square=\mathbf{1} \oplus \mathbf{3}, \quad \square=1 \oplus \mathbf{3} \oplus(\mathbf{5} \oplus \mathbf{1}), \square=2 \times \mathbf{5} \oplus 3 \times \mathbf{3} \oplus \mathbf{1}, \\
& \square=3 \times \mathbf{5} \oplus \mathbf{3} \oplus 2 \times \mathbf{1}, \quad \square=2 \times \mathbf{3},
\end{aligned}
$$

where the representation of dimension $2 s+1$ corresponds to a particle of spin $s$.

Thus a general superfield decomposes into the representations obtained in the process above, and corresponds to a given representation of the complexification of the algebra (2.3). It turns out that this representation is reducible. Furthermore, as it is the case in usual supersymmetry, it should certainly be interesting to construct constraint superfields in order to obtain various models invariant under equations (2.3). Having the decomposition of the field $\Phi$ into the monomials in $\theta$, and using (3.10), we are in principle able to obtain the transformation properties for the various components of $\Phi$.

At this point, an interesting additional possibility to construct slightly different superfields emerges naturally. It is well known that a physically important representation of the parafermionic algebra exists, namely the Green ansatz given by

$$
\theta^{M}=\frac{1}{\sqrt{2}}\left(\theta_{(1)}^{M}+\theta_{(2)}^{M}\right), \quad \partial_{M}=\frac{1}{\sqrt{2}}\left(\partial_{M}^{(1)}+\partial_{M}^{(2)}\right)
$$

\footnotetext{
${ }^{14}$ In what follows, the letters $M, N=0, \cdots, 4$ correspond to the 5 -dimensional indices and we have identified $\theta=\theta^{4}$.
} 
such that following relations are satisfied:

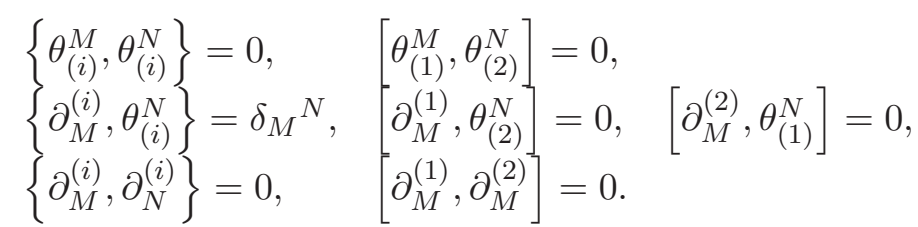

This means that fermions in the same subspace anticommute, while fermions in different subspaces commute. The normalisation in (4.5) is chosen to ensure that the relations (4.6) reproduce equations (3.3) and (3.7). Introducing fermionic oscillators $\left(\eta^{M}, \partial_{\eta^{M}}\right)$, the Green ansatz can be reformulated as

$$
\theta^{M}=\frac{1}{\sqrt{2}}\left(\eta^{M} \otimes 1+1 \otimes \eta^{M}\right), \quad \partial_{M}=\frac{1}{\sqrt{2}}\left(\partial_{\eta^{M}} \otimes 1+1 \otimes \partial_{\eta^{M}}\right) .
$$

It is important to emphasise that, with the representation (4.6), we gain quadratic relations between the paragrassmann variables and their derivatives. This implies in particular that

$$
\partial_{\mu}\left(\theta^{\nu}\right)=2 \delta_{\mu}^{\nu}, \quad \partial_{\mu}\left(\theta^{\nu} \theta^{\rho}\right)=2 \delta_{\mu}^{\nu} \theta^{\rho}, \quad \partial_{1}\left(\left(\theta^{2}\right)^{2} \theta^{1}\right)=-2\left(\theta^{2}\right)^{2},
$$

etc., holds. Within the Green ansatz, the supercharges then become

$$
V=\varepsilon_{i}^{\mu} \partial_{\mu}^{i}-\left(\theta_{2} \varepsilon_{1}^{\mu}+\theta_{1} \varepsilon_{2}^{\mu}\right) \theta_{1} \cdot \theta_{2} P_{\mu} .
$$

It is obvious that an arbitrary monomial in the paragrassmann variables can be written as a monomial in its Green component, but the converse is of course generally wrong. The precise relation between these two types of polynomials have been studied in detail in [4. As a consequence, the set of polynomials in the Green components is larger than the set of polynomials in the paragrassmann variables. This opens the possibility to define an "extended" superfield which depends on the Green components of the parafermions $\theta$ instead of $\theta$ itself:

$$
\tilde{\phi}\left(x^{\mu}, \theta_{1}^{M}, \theta_{2}^{M}\right) \text {. }
$$

Observe that this implies that the superfield (4.10) looks like an $N=2$ superfield. In this analogy, there are however some differences that should be observed carefully. Indeed, the variables $\theta_{i}^{M}$ are Lorentz vectors and $\theta_{1}^{M}$ commute with $\theta_{2}^{M}$. With this decomposition, we have the identity

$$
\tilde{\phi}\left(x^{\mu}, \theta_{1}^{M}, \theta_{2}^{M}\right)=\sum_{p_{1}, p_{2}=0}^{4} A_{\left[p_{1}, p_{2}\right] M_{1} \cdots M_{p_{1}} ; N_{1} \cdots N_{p_{2}}} \theta_{1}^{M_{1}} \cdots \theta_{1}^{N_{p_{1}}} \theta_{2}^{N_{2}} \cdots \theta_{2}^{N_{p_{2}}} .
$$

It should be taken into account that, in the decomposition above, the tensors $A_{\left[p_{1}, p_{2}\right]}$ are not in irreducible representations of $G L(1,4)$ (and a forciori of $S O(1,3)$ ). It is however not difficult to decompose the above product. Using the isomorphism between a $p$ - and an $(4-p)$-form, only a few products have to be identified. In contrast to the superfield (4.1), here we find that multiplicities 
for the component representations can be greater than one, i.e., we no more obtain multiplicity free reductions.

In any physical application, a central object in the construction of invariant Lagrangians is the covariant derivative, which commutes with $V$. Indeed, if an operator $D$ such that the condition $[D, V]=0$ is satisfied can be found, then the latter can be interpreted as a covariant derivative. This is a consequence of the Jacobi identity, which in connection with the considered operator $D$ implies the following relation:

$$
\delta[D, \Phi]=[Q,[D, \Phi]]=[D,[Q, \Phi]]=[D, \delta \Phi] .
$$

A routine but cumbersome computation shows that the construction of such a covariant derivative using (3.9) does not work. However, if we insist to obtain a covariant derivative, the obstructions can be surmounted by slightly modifying $V$ in equation (3.9). An admissible variation for this purpose is given if we define the corresponding operator as:

$$
\begin{aligned}
V & =\left[\epsilon^{\mu}, \partial_{\mu}\right]+\left(\left[\theta, \epsilon^{\mu}\right]\left[\theta_{\mu}, \theta^{\alpha}\right]+\left[\theta, \theta_{\mu}\right]\left[\varepsilon^{\mu}, \theta^{\alpha}\right]+\left[\theta, \theta^{\mu}\right]\left[\theta^{\mu}, \varepsilon^{\alpha}\right]\right) P_{\alpha}, \\
D & =[\theta, \partial]+\left[\theta, \theta^{\mu}\right]\left[\theta_{\mu}, \theta^{\alpha}\right] P_{\alpha},
\end{aligned}
$$

where $\partial$ is the "derivative" associated to the variable $\theta$. Now, using the relation $\left[\left[\theta^{\alpha}, \theta^{\beta}\right], \theta^{\gamma}\right]=0$, we finally get after some computation to the desired identity $[D, V]=0$. This fact enables us to define a constraint superfield as a superfield satisfying the condition

$$
\left[D, \Phi_{c}\right]=0 .
$$

Now observe that, since the $y^{\mu}$ are commuting variables and the conditions $\left[D, \theta^{\mu}\right]=0$ and $\left[D, y^{\mu}\right]=$ 0 for $y^{\mu}=x^{\mu}-\left[\theta, \theta^{\nu}\right]\left[\theta_{\nu}, \theta^{\mu}\right]$, the preceding commutator means that $\Phi_{c}$ takes a particularly simple expression

$$
\Phi_{c}\left(y^{\mu}, \theta^{\mu}\right)
$$

and does not depend explicitly on the variable $\theta$. This has the interesting consequence concerning the decomposition of the field (4.13) upon the rules given previously, namely, that only $G L(1,3)$ tensors have to be considered. Following this procedure it follows, in particular, that $\left\{\left|V_{1}, V_{2}, V_{3}\right|\right\}_{N} \cdot x^{\mu}$ vanishes identically. This means in particular that the cubic extension of the Poincaré algebra associated to (4.12) induces a space-time translation of a null vector. This point will be commented at the end of this section.

\subsection{Quaternary superfields}

The construction of quaternary superfields goes along the same lines as the construction of ternary superfields. We would like however to mention some interesting features. Consider the $D=10$ case. Looking at the supercharges given by (3.23) or (3.24), it is not difficult to see that a covariant derivative commuting with $Q_{A}$ (or with $Q$ ) cannot be found. However, as done already for the ternary case, a slight modification of equation (3.23) or (3.24) allows us to find operators that can be seen as covariant derivatives. We illustrate the procedure with paragrassmann variables. The key step is to introduce 


$$
\begin{aligned}
Q & =\left[\epsilon^{A}, \partial_{A}\right]+C_{-A B}\left[\theta^{A}, \theta^{B}\right]\left[\epsilon^{C}, \theta^{D}\right]\left(\Gamma^{M} C_{-}\right)_{C D} P_{M}-C_{-A B}\left[\epsilon^{A}, \theta^{B}\right]\left[\theta^{C}, \theta^{D}\right]\left(\Gamma^{M} C_{-}\right)_{C D} P_{M}, \\
D_{A} & =\partial_{A}-C_{-A B} \theta^{B}\left[\theta^{C}, \theta^{D}\right]\left(\Gamma^{M} C_{-}\right)_{C D} P_{M}+C_{-B C}\left[\epsilon^{B}, \theta^{C}\right] \theta^{D}\left(\Gamma^{M} C_{-}\right)_{D A} P_{M} .
\end{aligned}
$$

Using (3.3), a direct computation shows that $\left[D_{A}, Q\right]=0$ holds. Further, since the R.H.S of (4.14) involves commutators, we obtain that the action of $Q_{1} Q_{2} Q_{3} Q_{4}$ on the space-time vanishes because of the identity

$$
\left[Q_{1},\left[Q_{2},\left[Q_{2},\left[Q_{4}, x^{M}\right]\right]\right]\right]=0 .
$$

At this point we observe a quite interesting property (analogous to what happens in the cubic case) that arises at once from this consideration: the quartic extension of the Poincare algebra considered above induces a space-time translation of a null vector.

Another remarkable consequence of this modification concerns the algebraic structure of the extension. In contrast to the previously analyzed ternary case, here a $\mathbb{Z}_{2} \times \mathbb{Z}_{2} \times \mathbb{Z}_{2} \times \mathbb{Z}_{2}$-grading is enough to ensure the closure of the algebra via

$$
\left[Q_{1}, Q_{2}, Q_{3}, Q_{4}\right]=\sum_{\sigma \in S_{4}} \epsilon(\sigma) Q_{\sigma(1)} Q_{\sigma(2)} Q_{\sigma(3)} Q_{\sigma(4)}
$$

where $\epsilon(\sigma)$ denotes the signature of the permutation $\sigma$. With these specifications, we avoid completely the use of the complexification of the algebra.

Before closing this section, some comments are in order. As we have seen, if we impose the existence of a covariant derivative either in the cubic case or the quartic case, we automatically get that the higher degree extensions considered in this paper generate naturally a space-time translation of null vectors. Such a possibility is obviously excluded in usual supersymmetry. Indeed, recall that when we are studying massless representations (or massive representations with appropriate central charges, the so-called BPS-saturated states) half of the generators are inactive and generate a nilpotent subalgebra. The unitarity of the representation (absence of ghosts) forces us to represent the inactive charges by zero (see e.g. [31]). However, since we are considering here cubic and quartic algebras, it is not obvious at that the argument above remains valid. The consideration of higher order nilpotent extensions of the Poincaré algebra deserves further investigation. Let us mention that some kind of "nilpotent supersymmetry" in connection with the previous remark has already been considered, in the context of pure spinors and BRST symmetry (see [32] and references therein).

\section{Conclusions}

We have shown that parafermions are the relevant variables to construct an adapted superspace for higher order extensions of the Poincaré algebra (order two parafermions for cubic extensions and order three parafermions for quartic extensions). In particular this means that we were able to construct a differential realisation of the algebras (2.3) and (2.5) leading to an appropriate superspace. Among the quartic extensions, the $D=10$ case presents some interesting similarities with algebraic structures considered in supersymmetric theories [26].

For the classes of extensions considered, we have further analyzed the possibility of defining a covariant derivative, and have shown that such a fundamental object exits only if the algebra is "nilpotent" in the sense that it implies a space-time translation of null vector. This phenomenon 
is therefore deeply connected with the inner structure of the extension and superfields, and its range of validity has still to be explored. Although this point constitutes a fundamental difference when compared to the requirements of usual supersymmetry, we have to take into account that the transition to higher order extensions delivers new possibilities and structural properties, to which the usual phenomenological interpretations are no longer applicable automatically. For this reason, it cannot be inferred that the existence of such translations of null-vectors is intrinsically devoid of physical meaning.

Having identified the appropriate superfields associated to higher order extensions of the Poincaré algebra, one may wonder whether the standard techniques will be useful in the construction of physical models; since the product of superfields is a superfield, there is in principle no formal difficulty to construct an interacting theory.

Before closing this paper, let us make a final remark. As we have seen, parafermions become central for cubic and quartic extensions of the Poincaré algebra. In this context, the question whether this procedure can be generalized to higher order extensions arises at once. More specifically, one can ask whether the fully symmetric extensions of order $F$ (based upon Lie algebras of order $F$ ) also imply the possibility of constructing a differential realisation based on order $F-1$ parafermions operators. More generally, higher order extensions with fully antisymmetric brackets which can be seen as a special case of the colour algebras introduced in [12] can be considered. In a straight analogy one may wonder if parabosons would constitute the relevant variables in these cases.

To give an argument towards a positive answer to the last question, consider the algebra $I \mathfrak{s o}(1.3) \oplus\left\langle W_{\mu}, \mu=0, \cdots, 3\right\rangle$, that is, the Poincaré algebra together with Lorentz a vector $W_{\mu}$. Assume furthermore that the fully antisymmetric trilinear brackets between the operators $W$ close upon a space-time translation

$$
\left[W_{\mu}, W_{\nu}, W_{\rho}\right]=\epsilon_{\mu \nu \rho \sigma} P^{\sigma},
$$

with $[A, B, C]=A B C+B C A+C A B-A C B-B A C-C B A$ and satisfy the identity

$$
\left[W_{\mu},\left[W_{\nu}, W_{\rho}, W_{\sigma}\right]\right]-\left[\left[W_{\nu},\left[W_{\rho}, W_{\sigma}, W_{\mu}\right]\right]+\left[W_{\rho},\left[W_{\sigma}, W_{\mu}, W_{\nu}\right]\right]-\left[W_{\sigma},\left[W_{\mu}, W_{\nu}, W_{\rho}\right]\right]=0 .\right.
$$

This real algebra appears as a special case of the colour algebras introduced in [12]. Now all the results of section 3.1 can be applied directly with the following substitutions: the fully symmetric brackets $\{\cdots\}$ have to be substituted by the fully antisymmetric brackets $[\cdots]$ and commutators $[\theta, \partial],[\theta, \theta]$ etc by the anticommutators $\{\theta, \partial\},\{\theta, \theta\}$. For instance we have

$$
W=\left\{\varepsilon^{\mu}, \partial_{\mu}\right\}+\left\{\theta, \theta^{\mu}\right\}\left\{\varepsilon^{\sigma}, \theta_{\mu}\right\} P_{\sigma},
$$

for the supercharge. This means that the algebra (5.1)-(5.2) can be realised in terms of the order two parabosons $\theta, \partial$. However there is two differences compared to the algebra (2.3). Firstly there is no need to make a kind of "Jordan-Wigner" transformation since $\sum_{\sigma \in S_{3}} \epsilon(\sigma) W_{\sigma(1)} \cdot W_{\sigma(2)} \cdot W_{\sigma(3)} \cdot\left(\theta^{\alpha_{1}} \cdots \theta^{\alpha_{n}}\right)=0$. Secondly, although there is an analogous theorem for the decomposition of parabosons (see footnote 12) the corresponding superfield has an infinite number of degrees of freedom. Furthermore, it as to be noticed that this differential realisation induces a space-time translation of null vectors.

In conclusion, let us mention that the construction outlined here (see (5.1) and (5.2)), together with the results obtained in this paper, suggest that parafermions and parabosons could play 
some role in the description of higher order symmetries, but in a different context to its historical consideration and use.

\section{Acknowledgments}

During the preparation of this work, one of the authors (RCS) was financially supported by the research projects MTM2006-09152 of the M.E.C. and GR58/4120818-920920 of the UCM-BSCH.

\section{References}

[1] G. Gentile, Nuovo Cimento 17 (1940) 493.

[2] C. A. Nelson, J. Phys. A37 (2004) 2497.

[3] H. S. Green, Phys. Rev. 90 (1953) 270.

[4] Y. Ohnuki and S. Kamefuchi, Quantum Field Theory and Parastatistics Tokyo, Japan: Univ. Pr. (1982) Berlin, Germany: Springer (1982) 489p.

[5] O. W. Greenberg, Phys. Rev. Lett. 13 (1984) 598.

[6] O. W. Greenberg and A. M. L. Messiah, Phys. Rev. B136 (1964) 248; B138 (1964) 1155.

[7] S. Coleman and J. Mandula, Phys. Rev. 159 (1967) 1251; R. Haag, J. T. Lopuszanski and M. F. Sohnius, Nucl. Phys. B88 (1975) 257.

[8] J. Bagger and N. Lambert, Phys. Rev. D75 (2007) 045020 arXiv:hep-th/0611108].

[9] M. Rausch de Traubenberg, M. J. Slupinski, J. Math. Phys. 41 (2000) 4556 arXiv:hep-th/9904126]; M. Rausch de Traubenberg, M. J. Slupinski, J. Math. Phys. 43 (2002) 5145 arXiv:hep-th/0205113].

[10] M. Goze, M. Rausch de Traubenberg and A. Tanasa, J. Math. Phys. 48 (2007) 093507 arXiv:math-ph/0603008.

[11] M. Rausch de Traubenberg, J. Phys. Conf. Ser. 128 (2008) 012060 arXiv:0710.5368 [math-ph]]; M. Goze and M. Rausch de Traubenberg, J. Math. Phys. 50 (2009) 063508 arXiv:0809.4212 [math-ph]].

[12] R. Campoamor-Stursberg and M. Rausch de Traubenberg, J. of Generalized Lie Theory and Appl. 3 (2009) 113 arXiv:0811.3076 [math-ph]].

[13] N. Mohammedi, G. Moultaka and M. Rausch de Traubenberg, Int. J. Mod. Phys. A19 (2004) 5585 arXiv:hep-th/0305172.

[14] G. Moultaka, M. Rausch de Traubenberg and A. Tanasa, Int. J. Mod. Phys. A20 (2005) 5779 arXiv:hep-th/0411198.

[15] G. Moultaka, M. Rausch de Traubenberg and A. Tanasa, Proceedings of the XIth International Conference Symmetry Methods in Physics, Prague 21-24 June 2004 [arXiv:hep-th/0407168]. 
[16] C. Ahn, D. Bernard and A. Leclair, Nucl. Phys. B346 (1990) 409; S. Durand, Mod. Phys. Lett A8 (1993) 2323 hep-th/9305130; N. Fleury and M. Rausch de Traubenberg, Mod. Phys. Lett. A11 (1996) 899 hep-th/9510108]; A. Perez, M. Rausch de Traubenberg and P. Simon, Nucl. Phys. B482 (1996) 325 hep-th/9603149]; M. Rausch de Traubenberg and P. Simon, Nucl. Phys. B517 (1998) 485 hep-th/9606188.

[17] J. A. de Azcárraga and A. J. Macfarlane, J. Math. Phys. 37 (1996) 1115 hep-th/9506177); R. S. Dunne, A. J. Macfarlane, J. A. de Azcarraga and J. C. Perez Bueno, Phys. Lett. B 387 (1996) 294 arXiv:hep-th/9607220]; R. S. Dunne, A. J. Macfarlane, J. A. de Azcárraga and J. C. Pérez Bueno, Int. J. Mod. Phys. A12 (1997) 3275 hep-th/9610087.

[18] N. Roby, Bull. Sc. Math. 94 (1970) 49.

[19] I. Bars and M. Günäydin, J. Math. Phys. 20 (1979) 1977; I. Bars and M. Günäydin, Phys. Rev. D22 (1980) 1403; T. D. Palev, J. Math. Phys. 23 (1982) 1100; N. I. Stoilova and J. Van der Jeugt, Journal of Physics: Conference Series 128 (2008) 012061 arXiv:math-ph/0611085.

[20] J. Beckers and N. Debergh, Int. J. Mod. Phys. A8 (1993) 5041; A. G. Nikitin and V. V. Tretynyk, J. Phys. A28 (1995) 1655; J. Niederle and A. G. Nikitin, J. Phys. A32 (1999) 5141.

[21] M. Plyushchay, Int. J. Mod. Phys. A15 (2000) 3679 arXiv:hep-th/9903130]; S. Klishevich and M. Plyushchay, Mod. Phys. Lett. A14 (1999) 2739 [arXiv:hep-th/9905149]; F. Correa, V. Jakubsky, L. M. Nieto and M. S. Plyushchay, Phys. Rev. Lett. 101 (2008) 030403 arXiv:0801.1671 [hep-th]]; F. Correa, V. Jakubsky and M. S. Plyushchay, J. Phys. A41 (2008) 485303 [arXiv:0806.1614 [hep-th]].

[22] M. Rausch de Traubenberg, Pr. Inst. Mat. Nats. Akad. Nauk Ukr. Mat. Zastos., 50, Part 1, 2, 3, Natsional. Akad. Nauk Ukrani, Inst. Mat., Kiev, 2004, pp. 578 arXiv:hep-th/0312066.

[23] M. Rausch de Traubenberg, J. Phys. Conf. Series 175 (2009) 012003 [arXiv:0811.1465[hep-th]].

[24] V. Rittenberg and D. Wyler, J. Math. Phys. 19 (1978) 2193; V. Rittenberg and D. Wyler, Nucl. Phys. B139 (1978) 189; M. Scheunert, J. Math. Phys. 20 (1979) 712; H. S. Green and P. D. Jarvis, J. Math. Phys. 24 (1983) 1681; J. Lukierski and V. Rittenberg, Phys. Rev. D18 (1978) 385.

[25] R. Kerner, J. Math. Phys. 33 (1992) 403, V. Abramov, R. Kerner and B. Le Roy, J. Math. Phys. 38 (1997) 1650 arXiv:hep-th/9607143.

[26] I. C. G. Campbell and P. C. West, Nucl. Phys. B243 (1984) 112; M. Huq and M. A. Namazie, Class. Quant. Grav. 2 (1985) 293 [Erratum-ibid. 2 (1985) 597]; F. Giani and M. Pernici, Phys. Rev. D30 (1984) 325.

[27] M. Hamermesh, Group theory and its application to physical problems (Addison-Wesley, 1962); W. Fulton and J. Harris, Representation theory: a first course (Springer, 2004).

[28] Y. Ohnuki and S. Kamefuchi, Phys. Rev. 170 (1968) 1279.

[29] S. Kamefuchi and Y. Takahashi, Prog. Theor. Phys. (Kyoto) Suppl. 37 \& 38 (1966) 244.

[30] M. Rausch de Traubenberg, to appear in Adv. Appl. Cliff. Algebra arXiv:hep-th/0506011.

[31] J. Wess, J. Bagger, "Supersymmetry and Supergravity" (Princeton University Press, 1983).

[32] M. Cederwall, arXiv:0906.5490 [hep-th]. 